2 Beevers DG, Bloxham CA, Blackhouse CI, Lim CC, Watson RDS. The Remler M2,000 semiautomatic blood pressure recorder. Br Heart $\mathcal{f}$ $1979 ; 42: 366$.

${ }^{3}$ Rose GA, Holland WW, Crowley EA. A sphygmomanometer for epidemiologists. Lancet 1964 ; $1: 296-300$.

4 Masterton G, Main CJ, Lever AF, Lever RS. Low blood pressure in psychiatric inpatients. Br Heart $\mathcal{F} 1981 ; 45: 442-6$.

${ }^{5}$ Hunyor SN, Flynn JM, Cochíneas C. Comparison of performance of various sphygmomanometers with intra-arterial blood pressure readings. Br Med f 1978;ii:159-62.

(Accepted 14 September 1981)

Blood Pressure Clinic, Charitable Infirmary, Dublin 1, and Department of Clinical Pharmacology, Royal College of Surgeons in Ireland, Dublin 2

DESMOND J FITZGERALD, MRCPI, research fellow

KEVIN O'MALLEY, MD, FRCPI, professor of clinical pharmacology

EOIN T O'BRIEN, FRCPI, consultant physician (cardiology)

\section{Mask for continuous positive airway pressure: does it cause corneal abrasions?}

Severe pseudomonas panophthalmitis leading to the loss of an eye in an infant born at 32 weeks' gestation was recently reported from our neonatal unit. ${ }^{1}$ As pseudomonas conjunctivitis leads to the destruction of deeper eye tissues only in the presence of corneal damage ${ }^{23}$ we speculated that the facemask with which the baby was given continuous positive airway pressure for five days for treatment of hyaline membrane disease had caused such damage. We carried out a prospective study to test the hypothesis that the facemask used to give continuous positive airway pressure (Puritan-Bennet International Co-operation, Chichester) causes corneal abrasions.

\section{Patients, methods, and results}

We studied 79 preterm infants. Group 1 consisted of 18 infants with hyaline membrane disease who received respiratory support (continuous positive airway pressure or ventilation) by facemask. In accordance with our unit's policy the size of mask was chosen by the nursing staff so that it fitted comfortably over the infant's nose and mouth, producing minimal air leakage. The mask was held firmly in place by Netelast (Roussel Laboratories, London) and in most cases rested over the baby's eyes. Group 2 comprised 61 control infants, of whom 23 had mild hyaline membrane disease requiring treatment with only increased ambient oxygen. The remaining 38 babies had no respiratory problem.

After eight hours or more of continuous airway pressure the eyes of the infants in group 1 were stained with $1 \%$ fluorescein drops and the cornea examined under blue light. The eyes of babies in the control group were examined in the same way. All examinations were carried out in the first two

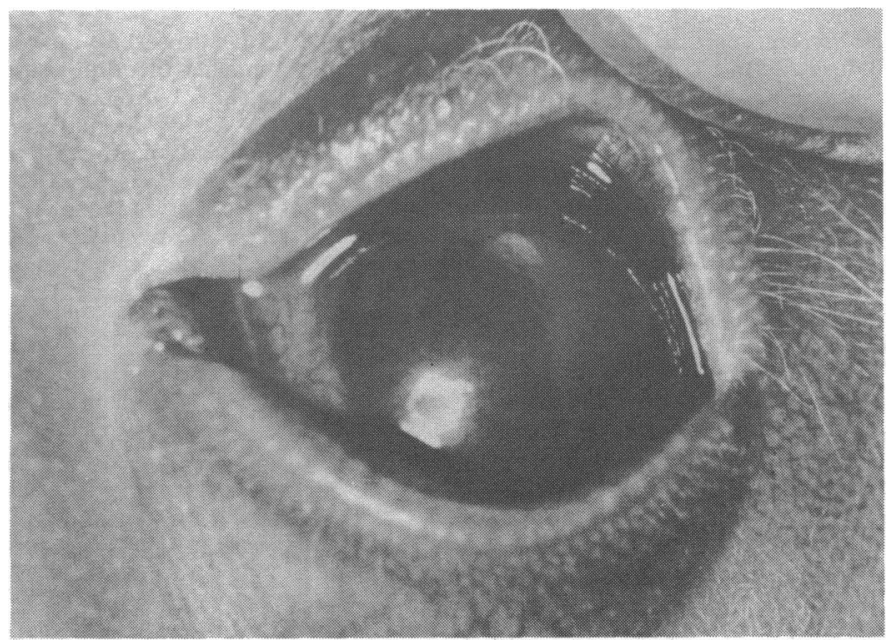

Corneal abrasion in an infant born at 28 weeks' gestation shown by fluorescein staining. days of life. When a corneal abrasion was suspected the eyes were reexamined by an ophthalmologist (PRC). Two corneal abrasions were found in the 18 infants treated with continuous positive airway pressure. The first was in the left cornea of a baby born at 28 weeks (birth weight $1230 \mathrm{~g}$ ), who was examined after 10 hours of continuous positive airway pressure (figure). The second abrasion occurred in an infant born at 32 weeks (birth weight $1560 \mathrm{~g}$ ), who was examined after 18 hours of continuous positive airway pressure. No corneal abrasions were found in the 61 control infants $(p=0.0497$, Fisher's exact test, significant at $5 \%$ level).

\section{Comment}

In our experience a small, well-fitting mask that does not encroach on the eyes commonly causes upper airways obstruction by pressing on the soft nose of infants. A larger mask that avoids this complication but rests on the eyes predisposes the baby to the risk of corneal abrasion. In our study the corneal abrasions caused by the facemask were transient, both healing completely within 24 hours of being detected. The potential danger of this iatrogenic hazard is that infection might establish itself and rapidly lead to panophthalmitis should the infant simultaneously develop conjunctivitis due to one of the proteusproducing organisms-for example, Pseudomonas, proteus. In a recent study $^{4} 4-5 \%$ of all cases of ophthalmia neonatorum were caused by Pseudomonas in babies on postnatal wards, and it would not be unreasonable to assume that the incidence might be higher in neonatal intensive care units.

Applying respiratory support by means of a facemask is a common neonatal practice, and this study has emphasised its small but important hazards. As a result of our findings we suggest that if sticky eyes develop when a baby is receiving ventilatory support via a facemask Gram staining as well as culture of the discharge should be requested. If Gram-negative organisms are found fluorescein examination of the cornea should be undertaken by an ophthalmologist. In the event of a corneal abrasion being present, topical, parenteral, and possibly subtenon ${ }^{5}$ antibiotics should be administered and alternative methods of ventilatory support used.

We thank Dr D P Davies, Dr K Simpson, and Dr P Swift, consultant paediatricians, for allowing us to study their patients.

1 Cole GF, Davies DP, Austin DJ. Pseudomonas ophthalmia neonatorum: a cause of blindness. $\mathrm{Br} \mathrm{Med} \mathcal{F} 1980 ; 281: 440-1$.

2 Ayliffe GAJ, Barry DR, Lowbury EJL, Roper-Hall MJ, Walker WM. Post-operative infection with Pseudomonas aeruginosa in an eye hospital. Lancet 1966;i :1113-7.

${ }^{3}$ Drewett SE, Payne DJH, Tuke W, Verdon PE. Eradication of Pseudomonas aeruginosa infection from a special care nursery. Lancet 1971 ; 946-8.

4 Armstrong JH, Zacarias F, Rein HF. Ophthalmia neonatorum: a char review. Pediatrics 1976;57:884-91.

5 Golden B, Coppel SP. Ocular tissue absorption of gentamicin. Arch Ophthalmol 1970;84:792-6.

(Accepted 7 September 1981)

\section{Leicester General Hospital, Leicester LE5 4PW}

GAYNOR F COLE, PHD, MRCP, paediatric senior registrar (present appointment : senior registrar, Queen Elizabeth Hospital for Children, London E2

Leicester Royal Infirmary, Leicester LE1 5WW

P RAY CHAUDHURI, FRCS, Do, senior registrar in ophthalmology

LIAM P CARROLL, MB, MRCP, paediatric senior registrar (present appointment : consultant paediatrician, Limerick, Ireland)

\section{Perverse $\mathbf{T}$ waves and chronic beta-blocker treatment}

T-wave inversion on the electrocardiogram is a non-specific abnormality that is also seen in some normal people, when its innocence may often be confirmed by its return to normal with beta-blockers. General recognition is needed that treatment with beta-blockers may itself cause $\mathrm{T}$-wave inversion.

\section{Patients, methods, and results}

Three men, aged 44-54, were investigated for chest pain, attributed ultimately to oesophageal spasm in two (cases 1 and 2) and a musculoskeleta cause in one (case 3 ). At presentation all patients were receiving chronic 
beta-blocker treatment (acebutolol $300 \mathrm{mg}$, atenolol $200 \mathrm{mg}$, and propranolol $160 \mathrm{mg}$ daily, respectively). In each patient $\mathrm{T}$-wave inversion was present in leads II, III, aVF, and V4-6: after exercise as well as at rest (supine and standing) in case 1 , only at rest in case 2 , and only after exercise in case 3 . Two weeks after beta-blocker treatment was stopped the $T$ waves became normally upright in each patient, though in case 1 they could again be inverted after the patient had been standing for 15 minutes.

Full cardiovascular investigation while the patients were not receiving any treatment included haemodynamic assessment, left ventricular and selective coronary angiography, arterial and coronary sinus lactate measurements, a pacing test, and provocation with ergometrine ( $500 \mu \mathrm{g}$ intravenously). There was no evidence of coronary artery stenosis, coronary artery spasm, myocardial ischaemia, or other cardiovascular abnormality. A maximal treadmill test $\left(\right.$ Bruce protocol ${ }^{2}$ ) was normal in all the patients.

The effects on the electrocardiogram of acute treatment with acebutolol $800 \mathrm{mg}$ by mouth over 12 hours (case 1 ) and propranolol $10 \mathrm{mg}$ intravenously (cases 2 and 3 ) were studied. The $T$ waves remained upright in each case both at rest (supine or standing) and on exercise testing, immediately and 20 minutes after exercise. A further course of chronic treatment with acebutolo $300 \mathrm{mg}$, atenolol $200 \mathrm{mg}$, and propranolol $320 \mathrm{mg}$ respectively for three weeks reproduced the original $\mathrm{T}$-wave inversion in cases 1 and 2 but not in case 3 (figure). After treatment was stopped again the $T$ waves became upright

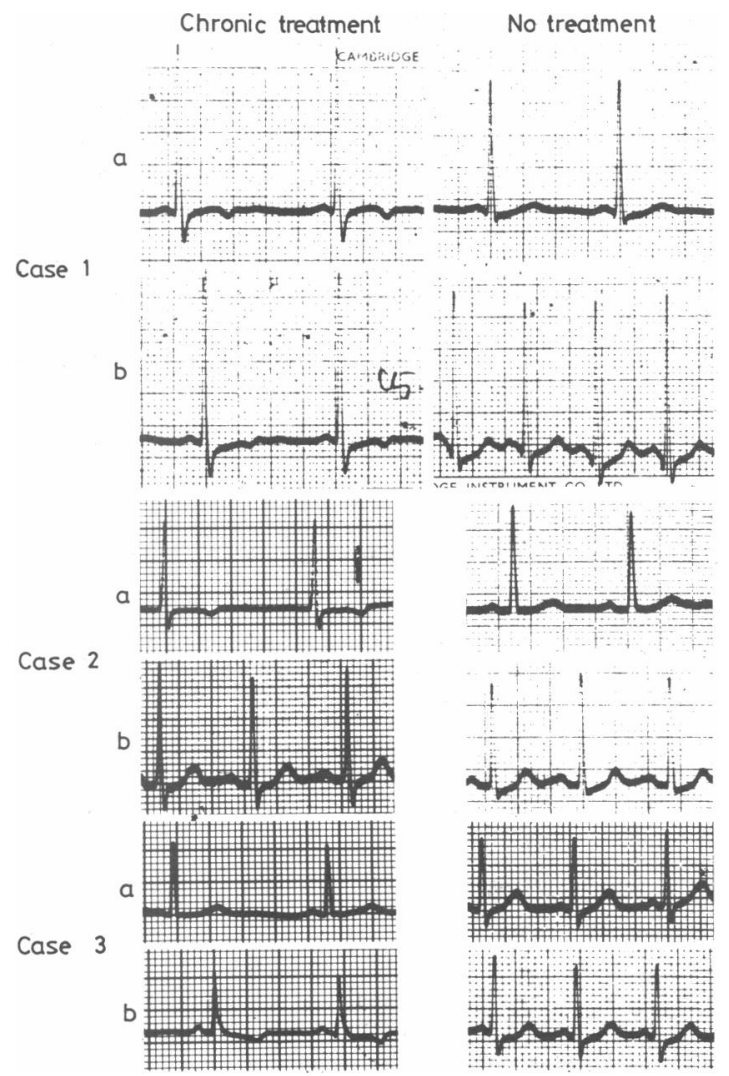

Electrocardiograms obtained in the three patients (supine, lead V5) before (a) and immediately after (b) exercise.

in all patients, though in case 1 they could again be inverted on standing. $T$ waves were not inverted in any patient by hyperventilation, but in case 1 hyperventilation normalised the inversion induced by standing when the patient was not receiving treatment.

\section{Comment}

Beta-blocker treatment has been thought to be a useful test for restoring to normal "innocent" T-wave inversion. ${ }^{3}$ Our observations indicate that chronic beta-blocker treatment may sometimes paradoxically be the cause of abnormal $\mathrm{T}$-wave inversion. This is perhaps not surprising, given that the direction of the $T$ wave is probably determined by slight regional differences in the relative duration of the action potential and that adrenergic activity influences their absolute duration and so may exert arbitrary effects on $\mathrm{T}$-wave direction. Chronic and acute beta-blocker treatment have different effects on the duration of action potential, ${ }^{4}$ which may also be relevant to our finding of $\mathrm{T}$-wave inversion with chronic but not acute treatment. Patients with non-cardiac chest pain are often inadvertently treated with beta- blockers. Unrecognised iatrogenic $\mathrm{T}$-wave inversion might add to the diagnostic difficulties in such patients.

1 Taggart P, Carruthers M, Joseph S, et al. Electrocardiographic changes resembling myocardial ischaemia in asymptomatic men with normal coronary arteriograms. Br Heart $\mathcal{f} 1979 ; 41: 214-25$.

${ }^{2}$ Bruce RA. Exercise testing of patients with coronary heart diseaseprinciples and normal standards for evaluation. Ann Clin Res 1971 ;3: 323.

3 Jackson WB. The use of propranolol in ECG diagnosis. NZ Med 7 1971; $73: 65-8$.

4 Raine AEG, Vaughan Williams EM. Electrophysiological basis for the contrasting prophylactic efficiency of acute and prolonged beta blockade. Br Heart $\mathcal{F} 1978 ; 40$, suppl:71-7.

(Accepted 11 September 1981)

Department of Cardiology, Welsh National School of Medicine, Cardiff CF4 4XN

T M GRIFFITH, MA, MRCP, registrar

J J DALAL, MD, lecturer (present address: cardiology division, Queen's University, Ontario, Canada)

W J PENNY, MB, MRCP, registrar

A M DART, BM, MRCP, registrar (present address: department of medicine, Woodend Hospital, Aberdeen)

A H HENDERSON, MB, FRCP, professor

\section{Insect-sting encephalopathy}

Cerebral infarction after insect stings occurs rarely. Extrapyramidal disturbances are extremely unusual, however, only two cases having been reported in which necrosis of the basal ganglia occurred. ${ }^{12} \mathrm{We}$ describe two patients in whom extrapyramidal syndromes developed after insect stings.

\section{Case reports}

CASE 1

A few minutes after being stung on the arm a 36-year-old nurse had a convulsion, after which blood pressure of $90 / 60 \mathrm{~mm} \mathrm{Hg}$ was recorded. She was given intravenous fluids, hydrocortisone, $10 \mathrm{mg}$ chlorpheniramine, and $12.5 \mathrm{mg}$ prochlorperazine intravenously. The next morning she complained of muscle aches; she had involuntary movements of the left leg, and her speech was soft and repetitive. She developed dystonia and her face became immobile. Increasing axial and limb rigidity with cogwheeling ensued. Tendon reflexes were brisk and plantar responses flexor. Cerebrospinal fluid was normal. A computed axial tomographic scan on the 12th day showed low attenuation in both posterior parietal regions.

Sequential treatment with dexamethasone, benzodiazepines, barbiturates, baclofen, dantrolene, benzhexol, and levodopa produced no benefit, and she developed generalised muscle spasms accompanied by rage, tachycardia, and sweating. After three months she had improved and could walk and feed herself.

CASE 2

Five minutes after being stung on the temple by a wasp a 38-year-old housewife lost consciousness and became cyanosed and her blood pressure became unrecordable. She was given parenteral adrenaline, hydrocortisone, and chlorpheniramine, and her blood pressure rose to $130 / 70 \mathrm{~mm} \mathbf{H g}$. During the subsequent hours she became restless and resisted attempts to move her limbs. There were slow, conjugate, roving eye movements, and oculocephalic movements were absent. She developed akinetic mutism and plastic hypertonicity in the limbs, and held either arm in any position in which it was placed for a few seconds. Tendon reflexes were symmetrically brisk, abdominal reflexes were absent, and both plantar responses were flexor. Cerebrospinal fluid was normal, and electroencephalography showed alpha rhythm and excess theta.

She improved slowly with dexamethasone, but her plantar responses became extensor and ankle clonus developed. There was no apparent visualfield defect. She showed some improvement and regained speech but required care in hospital. She died four months later from pulmonary embolism, but no further information became available.

\section{Comment}

Both patients suffered anaphylactic reactions to insect stings. Druginduced disturbances seem unlikely as, apart from a single dose of prochlorperazine administered to the first patient, neither received 